UDC: $519.234: 519.224$

43 (LXIX) 2 (2019), 61-72

DOI: $10.37560 /$ matbil2190061gb

\title{
PROPERTIES OF THE $k$-TH UPPER ORDER STATISTICS PROCESS THROUGH AN EXAMPLE
}

\author{
ANETA GACOVSKA-BARANDOVSKA
}

\begin{abstract}
The author has previously considered the asymptotic behavior of upper order statistics with central rank of a sample with deterministic size and of randomly indexed upper order statistics. In this paper, by using regular norming time-space changes, a theoretical example has been constructed in order to illustrate some of the obtained properties of the $k$-th upper order statistics process.
\end{abstract}

\section{INTRODUCTION}

Let $X_{1}, X_{2}, \ldots, X_{n}$ be a sample of independent identically distributed (iid) random variables (rvs) with common continuous distribution function (df) $F$. We denote $X_{1, n}=\max \left\{X_{i}\right\}, X_{2, n}=\max \left\{X_{i}\right\}$, for $L$ $i \in\{1,2, \ldots, n\} \quad i \in\{1,2, \ldots, n\} \backslash L$

the index of the first maximum, $\cdots, X_{n, n}=\min \left\{X_{i}\right\}$. The sequence $i \in\{1,2, \ldots, n\}$

$X_{n, n} \leq \ldots \leq X_{k, n} \leq \ldots \leq X_{1, n}$ is the ordered sample. The random variables $X_{n, n}, \ldots, X_{k, n}, \ldots, X_{1, n}$ are called upper order statistics and $X_{k, n}$ is the $k$-th upper order statistic (further on shortly written as u.o.s).

B.Vl. Gnedenko's paper (1943), "Sur la Distribution Limite du Terme Maximum d'une Serie Aleatoire", has marked the beginning of a new branch in the modern stochastics, the extreme value theory. He stated three limit distributions $\left(\Phi_{\alpha}, \alpha>0\right.$ - Fréchet, $\Psi_{\alpha}, \alpha>0$ - Weibull, $\Lambda$ - Gumbel) for the normalized maxima of iid random variables using linear normalization and gave the criteria for the domain of attraction. Since then, a lot of research has been done, both on the maxima and $k$-th order statistics. Smirnov, Leadbetter, Lindgren, Rootzen, Pancheva, Balkema, Embrechts, Kluppelberg, Mikosch and many more, have been working on the properties, the

2010 Mathematics Subject Classification. Primary: 62G20 Secondary: 62G30, 62E20.

Key words and phrases. Upper order statistic, fixed rank, central rank, regular norming sequence, random sample size, time-space changes. 
asymptotic theory under linear and monotone normalization, the limit distributions and their domain of attraction in cases of central and increasing rank ordered statistics. In Pancheva, Gacovska [9], the authors investigate the possible limit distributions of the $k$-th upper order statistics with central rank using monotone regular norming sequences and obtain thirteen possible types. In their further research, the authors investigated the asymptotic behavior of randomly indexed upper order statistics using regular norming time-space changes, Gacovska, Pancheva [5], and have looked into the possible application in reinsurance modeling. This paper illustrates some of the obtained properties through a theoretical example which gives basic directions on the further applications of ordered statistics theory in calculating reinsurance treaties.

\section{BAsic Model}

The theoretical background of the randomly indexed u.o.s. process has been completely described in Gacovska, Pancheva [5]. Here we give a short overview of the basic model, some properties are going to be proved, others just cited from previous papers. Never the less, we certainly need to introduce the terms and notations we use.

On a given probability space $(\Omega, \mathcal{A}, P)$, we use a point process $\mathcal{N}=$ $\left\{\left(T_{k}, X_{k}\right): k \geq 1\right\}$ defined in the following way:

i) The random arrival process $\left\{T_{k}\right\}$ consists of increasing time points $0<$ $T_{1}<T_{2}<\ldots<T_{n} \rightarrow \infty$. In order to use the results of the Central Limit Problem (C.L.P.), we suppose that the inter-arrival times $Y_{k}:=T_{k}-T_{k-1}$, $k \geq 1, T_{0}=0$ are independent rvs. Obviously $T_{n}=\sum_{k=1}^{n} Y_{k}$. The corresponding counting process $N(t):=\sum_{k} I\left\{T_{k} \leq t\right\}=\max \left\{k: T_{k} \leq t\right\}$ is right continuous.

ii) The random state process $\left\{X_{k}\right\}$ is built by positive iid rvs $X_{k}$ with continuous df $F_{X}$.

iii) Both sequences $\left\{T_{k}\right\}$ and $\left\{X_{k}\right\}$ are independent.

In this initial model, at every fixed moment $t>0$, we are supplied with a sample $\left\{X_{1}, X_{2}, \ldots, X_{N(t)}\right\}$ of random size $N(t)$. Our interest is focused on the u.o.s. of this sample

$$
X_{N(t): N(t)}<X_{N(t)-1: N(t)}<\ldots<X_{k: N(t)}<\ldots<X_{1: N(t)} .
$$

Definition 1. We call $Y_{k}: \Omega \times(0, \infty) \rightarrow(0, \infty), Y_{k}(t):=X_{k: N(t)}$ the $k$-th u.o.s. process. 
The asymptotic behavior of $Y_{k}(t)$ for $t \rightarrow \infty$ and $k$ fixed, under linear norming, is considered e.g in Embrechts, Kluppelberg, Mikosch [4, Chapter 4.3. For $k=1$ the process $Y_{1}(t)=\bigvee_{k=1}^{N(t)} X_{k}$ is an extremal process, taking into consideration for example Balkema, Pancheva [1] and Pancheva [10], where " $\bigvee$ " denotes the maximum operation. The extremal process $Y_{1}(\cdot)$ has independent max-increments $I(s, t]$ for all $0 \leq s<t$ and allows the decomposition $Y_{1}(t)=Y_{1}(s) \bigvee I(s, t]$. Consequently, as a solution of the C.L.P. one obtains the class of the max-infinitely divisible processes and its subclasses. Our process $Y_{k}(t)$ seems not to have the nice property of decomposition in independent components.

In this paper we illustrate the asymptotic behavior of the $k$-th u.o.s. process $Y_{k}(t)$ using monotone normalization. We assume that there exists a sequence of time-space changes on $(0, \infty) \times(0, \infty)$

$$
\zeta_{n}(t, x)=\left(\tau_{n}(t), u_{n}(x)\right),
$$

$\tau_{n}(t)$ and $u_{n}(x)$ continuous and strictly increasing, such that the normalized $k$-th u.o.s. process converges in law to a non-degenerate random process $Y^{(k)}(t)$, i.e.

$$
Y_{n}{ }^{(k)}(t):=u_{n}{ }^{-1} \circ Y_{k} \circ \tau_{n}(t)=u_{n}{ }^{-1}\left(X_{k: N\left(\tau_{n}(t)\right)}\right) \stackrel{d}{\longrightarrow} Y^{(k)}(t) .
$$

We also suppose that the norming sequence $\left\{\zeta_{n}\right\}$ is regular in the following sense: $\forall s>0$ there exist mappings $\mathcal{U}(s, x)$ and $\mathcal{T}(s, t)$, strictly increasing and continuous in $x$, respectively in $t$, such that for $n \rightarrow \infty$

$$
\begin{aligned}
& \lim _{n \rightarrow \infty} u_{[n s]}^{-1} \circ u_{n}(x)=\mathcal{U}(s, x) \\
& \lim _{n \rightarrow \infty} \tau_{[n s]}^{-1} \circ \tau_{n}(x)=\mathcal{T}(s, x) .
\end{aligned}
$$

Note that he mappings $s \rightarrow \mathcal{U}(s, \cdot)$ and $s \rightarrow \mathcal{T}(s, \cdot)$ are one-to-one. Usually we write $\mathcal{U}_{s}(x)$ and $\mathcal{T}_{s}(t)$ instead of $\mathcal{U}(s, x)$ and $\mathcal{T}(s, t)$.

The process $Y_{n}{ }^{(k)}(t)$ is connected to the point process

$$
\mathcal{N}_{n}=\left\{\left(T_{k, n}:=\tau_{n}^{-1}\left(T_{k}\right), X_{k, n}:=u_{n}^{-1}\left(X_{k}\right)\right): k \geq 1\right\}, n \geq 1 .
$$

Let $N_{n}(t)=\max \left\{k: T_{k, n} \leq t\right\}=\max \left\{k: T_{k} \leq \tau_{n}(t)\right\}=N\left(\tau_{n}(t)\right)$ be the counting process of $\mathcal{N}_{n}$. Let us consider the u.o.s. of the $n$-th sample series $\left\{X_{1, n}, X_{2, n}, \ldots, X_{N(t), n}\right\}$, namely

$$
X_{N_{n}(t): N_{n}(t), n}<\ldots<X_{k: N_{n}(t), n}<\ldots<X_{1: N_{n}(t), n}
$$

where $X_{k: N_{n}(t), n}=u_{n}^{-1}\left(X_{k: N\left(\tau_{n}(t)\right)}\right)=Y_{n}^{(k)}(t)$ is the $k$-th u.o.s. in the $n$-th sample series of size $N_{n}(t)$. In this way, with $n \rightarrow \infty$, the sample size $N_{n}(t)$ increases whereas the value of the state points $X_{k, n}$ decreases. 
For the limit process $Y^{(k)}(t)$ in $(2.3)$, which should be derived for certain samples, one should always be aware when does the limit distribution exist and which class it belongs to. The answers depend essentially on the character of the rank $k$. The results previously obtained take under consideration two separate cases: case 1 , when the rank $k$ is fixed, and case 2, when $k=k(n)$ is an increasing rank so that $\frac{k(n)}{n} \rightarrow \theta \in(0,1)$ (central order statistics).

In the following section, we answer the first question in the case of fixed rank, and mention results that characterize the limit distribution. The case of increasing rank has been completely considered in Gacovska, Pancheva [5]. Theorems 1 and 3, concern the asymptotic behavior of randomly indexed upper order statistics using regular norming time-space changes in both of the cases.

\section{RESUlts ON FiXed RANK CASE}

We return to the initial model of Section 2. Denote by $X_{k: n}$ the $k$ th u.o.s. of the sample $\left\{X_{1}, X_{2}, \ldots, X_{n}\right\}$ with a continuous df $F_{X}$. The necessary conditions for existence of a limit distribution of the normalized $X_{k: n}$ are given by:

Proposition 3.1. Suppose $F_{X} \in \max -\mathcal{D} \mathcal{A}$ of a max-stable $d f G$ with respect to a regular norming sequence $\left\{u_{n}(\cdot)\right\}$. Then for fixed $k$ and $n \rightarrow \infty$

$$
P\left(X_{k: n}<u_{n}(x)\right) \stackrel{w}{\longrightarrow} H(x)=\bar{\Gamma}_{k}(-\log G(x)),
$$

where $\Gamma_{k}(x)=\frac{1}{(k-1) !} \int_{0}^{x} t^{k-1} e^{-t} d t$ is the Gamma $d f$.

Proof. The proof of Proposition 3.1 is similar to the proof of Theorem 4.2.3 and Corollary 4.2.4 in Embrechts, Kluppelberg, Mikosch [4]. Let $F_{X}$ belong to the $\max -\mathcal{D} \mathcal{A}(G)$ with respect to a regular norming sequence $\left\{u_{n}\right\}$. It means that $n \bar{F}_{X}\left(u_{n}(x)\right) \stackrel{w}{\longrightarrow}-\log G(x)$. For fixed $x$ the the rv $B_{n}=\sum_{j=1}^{n} I\left\{X_{j} \geq u_{n}(x)\right\}$ is Binomial, i.e. $B_{n} \sim B i\left(n, \bar{F}_{X}\left(u_{n}(x)\right)\right)$. Observe that the event $\left\{B_{n}<k\right\}$ is equivalent to the event $\left\{X_{k: n}<u_{n}(x)\right\}$. Since

$$
E B_{n}=n p_{n}=n \bar{F}_{X}\left(u_{n}(x)\right) \stackrel{w}{\longrightarrow}-\log G(x),
$$

for $n \rightarrow \infty$, by the Poisson theorem, one obtains $B_{n} \stackrel{d}{\longrightarrow} P_{o}(\lambda), \lambda=-\log G(x)$.

Finally,

$$
\begin{aligned}
& P\left(X_{k: n}<u_{n}(x)\right)=P\left(B_{n}<k\right) \\
& \quad=\sum_{j=0}^{k-1} P\left(B_{n}=j\right)=\sum_{j=0}^{k-1}\left(\begin{array}{c}
n \\
j
\end{array}\right) \bar{F}^{j}\left(u_{n}(x)\right) F^{n-j}\left(u_{n}(x)\right)
\end{aligned}
$$




$$
\longrightarrow \sum_{j=0}^{k-1} \frac{(-\log G(x))^{j}}{j !} G(x) \triangleq \frac{1}{(k-1) !} \int_{-\log G(x)}^{\infty} t^{k-1} e^{-t} d t=\bar{\Gamma}_{k}(-\log G(x)) .
$$

The equality $\triangleq$ is a known result from mathematical analysis and can be easily obtained by multiple partial integration.

Before analyzing the limit class of the processes obtained in (2.3), we need to agree on the asymptotic behavior of the counting process $N_{n}(t)$. Let us assume that $\forall t>0$

$$
\frac{N_{n}(t)}{n} \stackrel{d}{\longrightarrow} \Lambda_{t}
$$

Here $\Lambda_{t}$ with df $F_{\Lambda}$ is random time change.

Definition 2. Under random time change we understand a strictly increasing and continuous random function $\Lambda:(0, \infty) \rightarrow(0, \infty), \Lambda(0)=0$ and $\Lambda(t) \rightarrow \infty$ for $t \rightarrow \infty$.

Next we formulate the first result on randomly indexed u.o.s.

Theorem 1. Suppose $F_{X} \in \max -\mathcal{D} \mathcal{A}(G)$ with respect to a regular norming sequence $\left\{u_{n}\right\}$. Assume that the counting process $N_{n}(t)$ satisfies (3.1). Then for fixed $k$ and $n \rightarrow \infty$

$$
P\left(X_{k: N_{n}(t)}<u_{n}(x)\right) \stackrel{w}{\longrightarrow} \int_{0}^{\infty} H(\mathcal{U}(s, x)) d F_{\Lambda(t)}(s)=E H \circ \mathcal{U}\left(\Lambda_{t}, x\right)
$$

where $H(x)=\bar{\Gamma}_{k}(-\log G(x))$ is the limit distribution from Proposition 3.1 $\operatorname{and} \mathcal{U}(s, x)=\lim _{n \rightarrow \infty} u_{[n s]}^{-1} \circ u_{n}(x)$.

In order to prove Theorem 1 we need the following analytical result:

Lemma 1. Assume $\left\{Q_{n}\right\}$ is a sequence of dfs on $(0, \infty)$ such that $Q_{n} \longrightarrow Q$ uniformly. Let $\left\{f_{n}\right\}$ be a sequence of functions on $(0, \infty)$ converging to $f$ $Q$-almost everywhere. Suppose $\left|f_{n}(z)\right| \leq 1$. Then $f$ is $Q$-integrable and

$$
\lim _{n \rightarrow \infty} \int_{0}^{\infty} f_{n}(z) d Q_{n}(z)=\int_{0}^{\infty} f(z) d Q(z) .
$$

Proof. $\left|\int_{0}^{\infty} f_{n}(z) d Q_{n}(z)-\int_{0}^{\infty} f(z) d Q(z)\right|$

$\leq\left|\int_{0}^{\infty} f_{n} d Q_{n}-\int_{0}^{\infty} f_{n} d Q\right|+\left|\int_{0}^{\infty} f_{n} d Q-\int_{0}^{\infty} f d Q\right|=I_{1}+I_{2} \rightarrow 0, n \rightarrow \infty$.

Namely, $I_{1} \leq \int_{0}^{\infty} d\left(Q_{n}-Q\right) \rightarrow 0$ due to the uniform convergence of $Q_{n}$ and $I_{2} \rightarrow 0$ using Lebesque's theorem for Dominated convergence. 
Proof of Theorem 1. Formally written, since $N_{n}(t)$ is integer valued, we have

$$
P\left(X_{k: N_{n}(t)}<u_{n}(x)\right)=\int_{0}^{\infty} P\left(X_{k: s}<u_{n}(x) \mid N_{n}(t)=s\right) d P\left(\frac{N_{n}(t)}{n}=\frac{s}{n}\right) .
$$

Substituting here $\frac{s}{n}=z$ and taking into account the property iii) of the initial model, we continue the above chain of equalities by

$=\int_{0}^{\infty} P\left(X_{k:[n z]}<u_{n}(x)\right) d P\left(\frac{N_{n}(t)}{n}=z\right)$.

The last integral is of the form $\int_{0}^{\infty} f_{n}(z) d Q_{n}(z)$, where for $n \rightarrow \infty$,

$Q_{n}(z):=P\left(\frac{N_{n}(t)}{n}<z\right)=\sum_{j=1}^{n z} P\left(N_{n}(t)=j\right)$

$\stackrel{w}{\longrightarrow} P\left(\Lambda_{t}<z\right)=F_{\Lambda_{t}}(z)=: Q(z)$,

$f_{n}(z):=P\left(X_{k:[n z]}<u_{n}(x)\right)=\sum_{j=0}^{k-1}\left(\begin{array}{c}{[n z]} \\ j\end{array}\right) \bar{F}^{j}\left(u_{n}(x)\right) F^{[n z]-j}\left(u_{n}(x)\right)$

$=P\left(X_{k:[n z]}<u_{[n z]}\left(u_{[n z]}^{-1} \circ u_{n}(x)\right)\right) \stackrel{w}{\longrightarrow} \bar{\Gamma}_{k}(-\log G(\mathcal{U}(z, x)))=: f(z), \forall z$.

Lemma 1 guarantees the convergence of $\int f_{n}(z) d Q_{n}(z) \longrightarrow \int f(z) d Q(z)$ on a set where the convergence $Q_{n} \longrightarrow Q$ is uniform. We observe that $Q_{n}(z)$ is continuous in $z$ and converges monotonically in $n$ to the continuous $Q(z)$. By Dini's theorem (see Rudin [11]), the convergence $Q_{n} \longrightarrow Q$ is uniform on compact subsets of $(0, \infty)$. Then for arbitrary $\delta, R>0$ and $\delta<R$, $\int_{\delta}^{R} f_{n} d Q_{n} \longrightarrow \int_{\delta}^{R} f d Q$, by Lemma 1 . Further we estimate

$$
\begin{aligned}
& \left|\int_{0}^{\delta} f_{n} d Q_{n}-\int_{0}^{\delta} f d Q\right|=\left|\int_{0}^{\delta} f_{n} d Q_{n}-\int_{0}^{\delta} f_{n} d Q+\int_{0}^{\delta} f_{n} d Q-\int_{0}^{\delta} f d Q\right| \\
& \leq\left|\int_{0}^{\delta} f_{n} d\left(Q_{n}-Q\right)\right|+\left|\int_{0}^{\delta} f_{n} d Q-\int_{0}^{\delta} f d Q\right|=I_{1}+I_{2} .
\end{aligned}
$$

Now $I_{1} \leq \int_{0}^{\delta} d\left(Q_{n}-Q\right) \rightarrow 0, n \rightarrow \infty$, because of the convergence $Q_{n} \longrightarrow Q$ and the fact that $Q$ does not have mass in 0 . Also $I_{2} \rightarrow 0$, due to the Dominated convergence theorem, and hence $\int_{0}^{\delta} f_{n} d Q_{n} \longrightarrow \int_{0}^{\delta} f d Q$. Just analogously, $\int_{R}^{\infty} f_{n} d Q_{n} \longrightarrow \int_{R}^{\infty} f d Q$. Consequently $\int_{0}^{\infty} f_{n}(z) d Q_{n}(z) \longrightarrow \int_{0}^{\infty} f(z) d Q(z)$.

Returning to the previous notations, for $n \rightarrow \infty$, we finally get the statement 


$$
\begin{aligned}
& \int_{0}^{\infty} P\left(X_{k:[n z]}<u_{n}(x)\right) d P\left(\frac{N_{n}(t)}{n}=z\right) \\
&= \int_{0}^{\infty} P\left(X_{k:[n z]}<u_{[n z]}\left(u_{[n z]}^{-1} \circ u_{n}(x)\right)\right) d P\left(\frac{N_{n}(t)}{n}=z\right) \\
& \stackrel{w}{\longrightarrow} \int_{0}^{\infty} H(\mathcal{U}(z, x)) d F_{\Lambda(t)}(z)=E H\left(\mathcal{U}\left(\Lambda_{t}, x\right)\right)=E \bar{\Gamma}_{k}\left(-\log G\left(\mathcal{U}\left(\Lambda_{t}, x\right)\right)\right) .
\end{aligned}
$$

Remark. In the asymptotic results for randomly indexed samples with size $N_{n}$, known in the literature, the authors usually suppose convergence in probability $\frac{N_{n}}{n} \stackrel{P}{\longrightarrow} \Lambda, \Lambda$ positive rv, e.g. Galambos [6], Theorem 6.2.1, Bilingsley [3], Theorem 17.2. In our model, we assume the sequences $\left\{N_{n}\right\}$ and $\left\{X_{n}\right\}$ are independent and $\Lambda$ is random time change. Those are the reasons why it is enough to suppose only convergence in distribution $\frac{N_{n}(t)}{n} \stackrel{d}{\longrightarrow} \Lambda_{t}$.

Corollary to Theorem 1 . Let $k=1$, then

$$
P\left(\bigvee_{j=1}^{N_{n}(t)} X_{j}<u_{n}(x)\right) \stackrel{w}{\longrightarrow} E G \circ \mathcal{U}\left(\Lambda_{t}, x\right)=E G^{\Lambda_{t}}(x) .
$$

Theorem 1 gives answer when the limit distribution exists, namely: If $F^{n}\left(u_{n}(x)\right) \stackrel{w}{\longrightarrow} G(x)$, where $\left\{u_{n}\right\}$ is regular, and if $\frac{N_{n}(t)}{n} \stackrel{d}{\longrightarrow} \Lambda_{t}, \Lambda_{t}$ random time change, then there exists a random process

$Y^{(k)}(t)$ with df $P\left(Y^{(k)}(t)<x\right)=: g(t, x)=E \bar{\Gamma}_{k}\left(-\log G\left(\mathcal{U}\left(\Lambda_{t}, x\right)\right)\right)$, such that

$Y_{n}^{(k)}(t)=u_{n}^{-1}\left(X_{k: N_{n}(t)}\right) \stackrel{d}{\longrightarrow} Y^{(k)}(t)$.

Using the regularity of the norming sequence $\left\{\tau_{n}\right\}$, the authors in Gacovska, Pancheva [5], prove Theorem 2 and classify the obtained limit process as self-similar process. Denote $\stackrel{f d d}{=}$ for equivalence of the finite dimensional distributions.

Theorem 2. The limit process $Y^{(k)}$ in (2.3) is self-similar with respect to the continuous one-parameter group $\left\{\eta_{s}(t, x)=\left(\mathcal{T}_{s}(t), \mathcal{U}_{s}(x)\right): s>0\right\}$ of time-space changes, i.e.

$$
Y^{(k)}(t) \stackrel{f d d}{=} \mathcal{U}_{s}^{-1} \circ Y^{(k)} \circ \mathcal{T}_{s}(t), \forall s>0 .
$$

When central u.o.s. process is considered, $Y_{n}^{\left(k_{n}\right)}(t)=u_{n}^{-1} \circ X_{k_{n}: N_{n}(t)}$, the rank $k=k_{n}$ increases with $n$ in such a way that

$$
\frac{k_{n}}{n} \rightarrow \theta \in(0,1)
$$


and

$$
\frac{N_{n}(t)}{n} \stackrel{d}{\longrightarrow} \Lambda_{t}
$$

$\Lambda_{t}$ random time change.

We ask for the asymptotic behavior of $Y_{n}^{\left(k_{n}\right)}(t)$ as $n \rightarrow \infty$. To this end, let us preliminarily consider a sample with non-random size $l_{n}, l_{n} \rightarrow \infty$ as $n \rightarrow \infty$, namely $\left\{X_{1}, X_{2}, \ldots, X_{l_{n}}\right\}$. We form the central order statistics (c.o.s.) $X_{k_{n}: l_{n}}$ with the property $\frac{k_{n}}{l_{n}} \rightarrow \lambda \in(0,1)$. As norming mappings we again use the regular sequence $\left\{u_{n}(\cdot)\right\}$ of space changes $u_{n}:(0, \infty) \rightarrow$ $(0, \infty)$. Denote $p_{n}(\cdot)=P\left(X_{i} \geq u_{n}(\cdot)\right)$. Just analogously to Theorem 2.5.2 in Leadbetter, Lindgren, Rootzen [7], where $u_{n}(x)$ are linear, one can prove the following statement:

Proposition 3.2. Suppose $k_{n} \rightarrow \infty, k_{n}<l_{n}, \frac{k_{n}}{l_{n}} \rightarrow \lambda \in(0,1)$ and $l_{n}(1-$ $\left.p_{n}(\cdot)\right) p_{n}(\cdot) \rightarrow \infty$, as $n \rightarrow \infty$. Let

$$
\frac{k_{n}-l_{n} p_{n}(x)}{\sqrt{l_{n} p_{n}(x)\left(1-p_{n}(x)\right)}} \stackrel{w}{\rightarrow} \tau_{\lambda}(x) .
$$

Then for $n \rightarrow \infty$

$$
P\left(X_{k_{n}: l_{n}}<u_{n}(x)\right) \stackrel{w}{\longrightarrow} \Phi\left(\tau_{\lambda}(x)\right)
$$

where $\Phi(x)$ is the standard normal $d f$.

Sometimes we use the notation $\tau(\lambda, x)$ instead of $\tau_{\lambda}(x)$. In order to obtain a unique limit distribution in (3.6), usually one assumes the second order condition $\sqrt{n}\left(\frac{k_{n}}{l_{n}}-\lambda\right) \rightarrow 0$, for $n \rightarrow \infty$.

An equivalent version of Proposition 3.2 is proved in Pancheva, Gacovska [9]. Moreover, there it has been shown that $\tau_{\theta}$ satisfies the functional equation

$$
\sqrt{z} \cdot \tau(\theta, x)=\tau\left(\theta, \mathcal{U}_{z}(x)\right)
$$

$\forall z>0, x$ continuity points of $\tau$ and $\mathcal{U}_{z}(x)$ from (2.4a). As a consequence one deduces that the limit class in $(3.6)$ contains thirteen possible types.

More results on the asymptotic behavior of randomly indexed ordered statistics can be found in Barakat, El-Shandidy [2] and in Surkov [12].

For the randomly indexed c.o.s. $X_{k_{n}: N_{n}(t)}$ satisfying (3.1) and (3.4), using the previous Proposition 3.2, Gacovska, Pancheva [5] stated the main result and give proofs to the following two theorems:

Theorem 3. Suppose $k_{n} \rightarrow \infty$ and (3.4) is satisfied. Assume there exists a regular norming sequence $\left\{u_{n}(\cdot)\right\}$ such that $n \bar{F}\left(u_{n}(x)\right) F\left(u_{n}(x)\right) \rightarrow \infty$ and

$$
\sqrt{n} \frac{\theta-\bar{F}\left(u_{n}(x)\right)}{\sqrt{\theta(1-\theta)}} \stackrel{w}{\longrightarrow} \tau_{\theta}(x),
$$


for $n \rightarrow \infty$. If additionally (3.1) holds true, then $\forall t>0$

$$
u_{n}^{-1} \circ X_{k_{n}: N_{n}(t)} \stackrel{d}{\longrightarrow} Y_{0}(t)
$$

where the limit process has df

$g_{0}(t, x)=\int_{\theta}^{\infty} \Phi \circ \tau\left(\frac{\theta}{z}, \mathcal{U}_{z}(x)\right) d F_{\Lambda(t)}(z)=E\left[\Phi \circ \tau\left(\theta \Lambda_{t}^{-1}, \mathcal{U}_{\Lambda(t)}(x)\right) I\left\{\Lambda_{t}>\theta\right\}\right]$ and $\mathcal{U}(\cdot, \cdot)$ is defined in 2.4a).

Theorem 3 provides the conditions for existance of the limit distribution of the $k$-th u.o.s. process, in the case of increasing rank $k_{n}$. The authors also characterize the limit process $Y_{0}$ as self-similar. A nice consequence of the selfsimilarity of a random process $Y$, i.e. $\mathcal{U}_{s} \circ Y(t) \stackrel{d}{=} Y \circ \mathcal{T}_{s}(t)$, is the possibility to determine its fdd's knowing only the df $g(1, x):=P(Y(1)<x)$. Denote by $s(t)$ the unite solution of $\mathcal{T}_{s}(t)=1$. Consequently $Y(t) \stackrel{d}{=} \mathcal{U}_{s(t)}^{-1} \circ Y(1)$, $\forall t>0$. Then

$$
P\left(Y\left(t_{1}\right)<x_{1}, \ldots, Y\left(t_{k}\right)<x_{k}\right)=P\left(Y(1)<\min \left(\mathcal{U}_{s\left(t_{1}\right)}\left(x_{1}\right), \ldots, \mathcal{U}_{s\left(t_{k}\right)}\left(x_{k}\right)\right)\right) .
$$

\section{EXAmple}

To stress out the importance of application of the basic model in concrete cases, mostly in insurance mathematics, hereby we construct a specific example. To make it closer to practise, we specify the initial model enriched by some additional assumptions:

For i): We additionally assume that the inter-arrival times $\left\{Y_{k}\right\}, Y_{k}=$ $T_{k}-T_{k-1}$, are iid rvs which df $F_{Y}$ has a regularly varying tail, namely $\bar{F}_{Y} \in R V_{-\beta}, \beta \in(0,1)$. Consequently, by the stable CLT there exists a sequence $\{b(n)\}, b(n)>0$, such that

$$
\frac{T_{n}}{b(n)}=\sum_{k=1}^{n} \frac{Y_{k}}{b(n)} \stackrel{d}{\longrightarrow} \mathcal{D}_{\beta}
$$

where $\mathcal{D}_{\beta}$ is a $\beta$-stable rv. Then $\forall t>0$, by the functional stable CLT,

$$
\mathcal{D}_{n}(t):=\frac{T_{[n t]}}{b(n)}=\sum_{k=1}^{[n t]} \frac{Y_{k}}{b(n)} \stackrel{d}{\longrightarrow} \mathcal{D}(t), \mathcal{D}(1) \stackrel{d}{=} \mathcal{D}_{\beta}
$$

Here $\mathcal{D}(t)$ is an one-sided $\beta$-stable random process. 
The counting process $N(t)$ associated with the arrival time process $\left\{T_{n}\right\}$ is determined by

$$
N(t)=\max \left\{n: T_{n} \leq t\right\}=\max \left\{n: \sum_{k=1}^{n} \frac{Y_{k}}{b(n)} \leq \frac{t}{b(n)}\right\} .
$$

Let us denote by $E(t)$ the inverse process to $\mathcal{D}(t)$, the so called hitting time process for $\mathcal{D}(t)$ :

$$
E(t)=\inf \{s: \mathcal{D}(s)>t\} .
$$

Now the equivalence of the random events

$$
\{N(t) \geq n\} \Longleftrightarrow\left\{T_{n} \leq t\right\}
$$

implies the equivalence

$$
\{\mathcal{D}(x) \geq t\} \Longleftrightarrow\{E(t) \leq x\} .
$$

Denote by $\widetilde{b}(n)$ the asymptotically inverse sequence to $b(n)$ in the sense that

$$
b(\widetilde{b}(n)) \sim n
$$

Since $T_{n}=\sum_{k=1}^{n} Y_{k}$ is strictly increasing, one takes ad hoc $b(n)$ strictly increasing in $n$, too. Thus, $\widetilde{b_{n}}(b(n)) \sim n$ is also true. In view of 4.2), 4.3 and 4.4 one may conclude that

$$
\frac{N(n t)}{\widetilde{b}(n)} \stackrel{d}{\longrightarrow} E(t)
$$

Indeed:

$$
\begin{aligned}
& P\left(\frac{N(n t)}{\widetilde{b}(n)}<x\right)=P(N(n t)<\widetilde{b}(n) x)=P\left(T_{[\widetilde{b}(n) x]}>n t\right) \\
& =P\left([b(\widetilde{b}(n))]^{-1} \cdot T_{[\widetilde{b}(n) x]}>[b(\widetilde{b}(n))]^{-1} \cdot n t\right) \\
& \stackrel{w}{\longrightarrow} P(\mathcal{D}(x)>t)=P(E(t)<x)=F_{E(t)}(x) .
\end{aligned}
$$

Here the limit process $E(t)$ is a $\beta$-self-similar process in the sense that

$$
E(c t) \stackrel{f d d}{=} c^{\beta} E(t), \quad \forall c>0 .
$$

This and other properties of the hitting time process $E(t)$ are studied in Meerschaert, Scheffler [8]. Note that (4.5) is equivalent to

$$
\frac{N(b(n) t)}{n} \stackrel{d}{\longrightarrow} E(t) \text {. }
$$

Here $E(t)=t^{\beta} E(1)$ is stochastically continuous and strictly increasing. The last limit relation is nothing else but condition (3.1) in the previous section. Thus, in the transformed model $\mathcal{N}_{n}$ we are left to choose $\tau_{n}(t)=b(n) t$. 
For ii) we additionally assume that the state points $X_{k}$ are iid, standard Pareto distributed rvs, i.e. $F_{X}(x)=1-\frac{1}{x}, x \geq 1$. This assumption brings the example closer to practise. The Pareto tailed claims are regularly used in insurance for modeling reinsurance treaties.

For iii) there are no additional assumptions.

Next we have to determine the space changes $u_{n}(x)$ for the transformed model $\mathcal{N}_{n}=\left\{\left(T_{k n}, X_{k n}\right): k \geq 1\right\}$. In the Pareto case, Proposition 3.2 says that for $l_{n}=n, \frac{k_{n}}{n} \rightarrow \theta \in(0,1)$ and $n \rightarrow \infty$,

$$
P\left(X_{k_{n}: n}<u_{n}(x)\right) \stackrel{w}{\longrightarrow} H(x)=\Phi\left(\tau_{\theta}(x)\right) \text { if } \sqrt{n} \cdot \frac{\theta-1 / u_{n}(x)}{\sqrt{\theta(1-\theta)}} \stackrel{w}{\longrightarrow} \tau_{\theta}(x) .
$$

Let us consider the particular case where $H(x)$ is continuous and strictly increasing on its support. Then $\tau_{\theta}$ satisfies the functional equation $\tau_{\theta}^{-1}\left(\sqrt{z} \cdot \tau_{\theta}(x)\right)=\mathcal{U}_{z}(x)$ (equivalent to $(3.7)$ ), where $\mathcal{U}_{z}$ is the limit mapping in 2.4a). We may take $\tau_{\theta}(x)=\sqrt{\frac{x}{\theta(1-\theta)}}$, for $x \in \operatorname{supp} H$ and observe that $\theta-\frac{1}{u_{n}(x)} \sim \sqrt{\frac{x}{n}}$. This suggests to determine the space changes as $u_{n}(x)=$ $\frac{\sqrt{n}}{\theta \sqrt{n}-\sqrt{x}}$. Doing so, we obtain $u_{n}^{-1}(x)=n\left(\frac{1}{x}-\theta\right)^{2}$ and $u_{[n z]}^{-1} \circ u_{n}(x)=$ $\frac{[n z]}{n} x \rightarrow z x=\mathcal{U}_{z}(x)$. Thus, $P\left(X_{k_{n}: n}<u_{n}(x)\right) \stackrel{w}{\longrightarrow} \Phi\left(\sqrt{\frac{x}{\theta(1-\theta)}}\right)$.

We apply the last result to randomly indexed c.o.s. in the transformed model $\mathcal{N}_{n}$ :

$$
\begin{aligned}
& P\left(X_{k_{n}: N_{n}(t)}<u_{n}(x)\right)=P\left(X_{k_{n}: N(b(n) t)}<u_{n}(x)\right) \\
& =\int_{0}^{\infty} P\left(X_{k_{n}: N(b(n) t)}<u_{n}(x) \mid N(b(n) t)=s\right) d P\left(\frac{N(b(n) t)}{n}=\frac{s}{n}\right) \\
& \stackrel{w}{\longrightarrow} \int_{\theta}^{\infty} \Phi\left(\tau\left(\frac{\theta}{z}, U_{z}(x)\right)\right) d F_{E(t)}(z) .
\end{aligned}
$$

Since in our model $\tau\left(\frac{\theta}{z}, U_{z}(x)\right)=\sqrt{\frac{z^{3} x}{\theta(z-\theta)}}$, we can continue with

$$
=\int_{\theta}^{\infty} \Phi\left(\sqrt{\frac{z^{3} x}{\theta(z-\theta)}}\right) d F_{E(t)}(z)=g_{0}(t, x)=P\left(Y_{0}(t)<x\right) .
$$

In this way, the univariate distributions $g_{0}(t, x)$ of the limit process are being determined.

At the end, let us discuss the asymptotic character of the space changes $u_{n}(x)$ :

a) Assume $k$ is fixed. The properties of $\left\{u_{n}(x)\right\}$ are a consequence of the condition $F_{X} \in \max -\mathcal{D} \mathcal{A}(G)$, i.e. $\bar{F}_{X}\left(u_{n}(x)\right) \sim-\frac{1}{n} \log G(x)$. Then for $n \rightarrow \infty, u_{n}(x) \longrightarrow r_{F}$, where $r_{F}$ is the right end point of the $\operatorname{supp} F_{X}$.

b) Assume $k=k_{n}$ increases to $\infty$ in such a way that $\frac{k_{n}}{n} \rightarrow \theta \in(0,1)$. In this case the properties of $\left\{u_{n}(x)\right\}$ are determined by condition (14). Then $F_{X} \notin \max -\mathcal{D} \mathcal{A}$ of any max-stable df and $\theta-\bar{F}_{X}\left(u_{n}(x)\right) \longrightarrow 0$. Hence, $u_{n}(x) \longrightarrow x_{1-\theta}$, the $(1-\theta)$ quantile of $F_{X}$. 


\section{REFERENCES}

[1] A. A. Balkema, E. Pancheva, Decomposition for multivariate extremal processes, Commun. Statist.-Theory Methods, 25-4 (1996), 737-758.

[2] H. M. Barakat, M. A. El-Shandidy, On general asymptotic behavior of order statistics with random index, Bull. Malays. Math. Sci. Soc., 27 (2) (2004), 169-183.

[3] P. Billingsley, Convergence of Probability Measures, 2nd Edition, John Wiley and Sons, Inc., 1999.

[4] P. Embrechts, C. Kluppelberg, T . Mikosch, Modelling Extermal Events for Insurance and Finance, Heidelberg, Springer, 1997.

[5] A. Gacovska, E. Pancheva, Randomly indexed central order statistics, C. R. Acad. Bulg. Sci., 66 (7) (2013), 951-960.

[6] J. Galambos, The Asymptotic Theory of Extreme Order Statistics, John Wiley and Sons, Inc., 1984.

[7] M. R. Leadbetter, G. Lindgren, H. Rootzen, Extremes and Related Properties of Random Sequences and Processes, Springer, 1983.

[8] M. M. Meerschaert, H. P. Scheffler, Limit theorems for continuous-time random walks with infinite mean waiting times, J. Appl. Prob., 41 (2004), 623-638.

[9] E. Pancheva, A. Gacovska, Asymptotic behaviour of central order statistics under monotone normalization, Theory Probab. Appl., 58 (1) (2013), 177-192, russian edition.

[10] E. Pancheva, Self-similar extremal processes, Journal of Mathematical Sciences, Plenum Publishing Corporation, New York, 92 (3) (1998), 3911-3920.

[11] W. Rudin, Principles of Mathematical Analysis, 3rd Edition, Mc-Graw Hill, 1976.

[12] A. Surkov, On the asymptotic behavoir of the intermediate order statistics with random size of a sample, Journal of Math. Sci., 127 (4) (2005), 2082-2090.

Aneta Gacovska-Barandovska

Ss. Cyril and Methodius University,

Faculty of Natural Sciences and Mathematics,

Arhimedova 3, 1000 Skopje,

North Macedonia

E-mail address: aneta@pmf.ukim.mk, agacovska@gmail.com 